\title{
Actitudes hacia la discapacidad de jóvenes y adultos de Chiapas
}

Attitudes towards disability in youths and adults of Chiapas

Recibido: mayo 17 de 2009 | Revisado: octubre 26 de 2009 | Aceptado: mayo 2 de 2010

\author{
Germán Alejandro García Lara* \\ Universidad Pedagógica Nacional, Chiapas, México \\ SOLEDAD HERNÁNDEZ ORTIZ ** \\ Instituto de Estudios Superiores de Chiapas, México
}

Para Alan Ernesto

SICI: 1657-9267(201112)10:3<817:AHDJAC>2.3.TX;2-Q

Para citar este artículo. García, L. G. A. \& Hernández, O. S. (2011). Actitudes hacia la discapacidad de jóvenes y adultos de Chiapas. Universitas Psychologica, 10 (3), 817-827.

Unidad 071 Tuxtla Gutiérrez, Chiapas, México. E-mail: asiaaa@hotmail.com

** Blvd. Paso León No. 244, Tuxla Gutiérrez, Chiapas, México.E-mail: sola1221.iraaa@hotmail.com

\section{RESUMEN}

El estudio se realiza en una población de 736 jóvenes y adultos de tres localidades de Chiapas, México, y tiene como objetivo analizar las actitudes que estos presentan sobre la discapacidad. Se aplicaron la Escala de Valoración de Términos Asociados con la Discapacidad y la Escala de Actitudes hacia las Personas con Discapacidad, Forma G. Los resultados indican que las variables: tener un familiar con discapacidad, poseer una mayor escolaridad, ser más joven, soltero o casado, vivir en zona urbana y no profesar ninguna religión, señalan actitudes más positivas hacia estas personas. Se da una valoración más positiva a los términos con necesidades educativas especiales, excepcional y enfermo, y más negativa a subnormal, trastornado y retrasado.

Palabras clave autores

Actitudes, discapacidad.

Palabras clave descriptores

Pruebas psicológicas, personalidad, investigación cuantitativa.

\section{A B S T R A C T}

The study is realized in a population of 736 young men and adults of three Chiapas's localities, México and it has as aim analyze the attitudes that these present on the disability. There are applied the Scale of Valuation of Terms Associated with the Disability and the Scale of Attitudes towards the Persons by Disability, Form G. The results indicate that the variables: to have a relative with disability, a major education, the youngest, single or married being, to live in urban zone and, not to practice any religion, they indicate more positive attitudes towards these persons. One gives a more positive valuation to the terms with educational special needs, exceptional and sick, and more negative to subnormal, upset and delayed.

Key words authors

Attitudes, disability.

Key words plus

Psychologycal test, personality, quantitative research. 


\section{Introducción}

El concepto de actitud surge en los albores del siglo XX, para denominar ciertas predisposiciones de los individuos tendientes a la evaluación de determinados objetos y actuar en correspondencia con esas apreciaciones. Aunque las actitudes se equiparan con los valores en términos de guía u orientación de la conducta de los sujetos, las actitudes hacen referencia a "los aspectos más periféricos (somáticos) y observables de los afectos (...), por el contrario, los valores, en tanto que apreciación cognitivo evaluativa del sentimiento pueden ser eventualmente considerados como propiedades al margen de los afectos que las relaciones con lo que ellos nos inspiran" (Asensio, 2002, p. 57). De ello deviene que la inclusión de juicios o valoraciones, son aspectos que aún se discute si deben o no ser incorporados al análisis de las actitudes.

De acuerdo con Parasuram (2006), el concepto de actitud es probablemente el más distintivo en la Psicología Social contemporánea. En el Diccionario de la Lengua Española (2001), la actitud es definida como "disposición de ánimo manifestada de algún modo" o "postura del cuerpo humano, especialmente cuando es determinada por los movimientos del ánimo". Esto, sin duda, orienta la definición a la afectividad de los sujetos.

Por su parte, el Diccionario de Psicología (Dorsch, 2005), define la actitud como una predisposición adquirida y estable para reaccionar ante una situación determinada. Como se aprecia, la referencia a predisposiciones y afecto son elementos asociados al concepto; asimismo, los aspectos centrales que dan cuenta de dicho término, hacen alusión al hecho de que la actitudes son adquiridas, implican una alta carga afectiva y emocional que refleja deseos, voluntad y sentimientos, y las que representan respuestas de carácter electivo, son aprendidas y configuradas como experiencias subjetivas y poseen dimensiones múltiples de expresión en los planos individual y social (Castro de Bustamante, 2002).

La relación entre cognición y afecto es evidente, si se acepta que aquello que se piensa acerca de un objeto influye decisivamente en los sen- timientos que estos despiertan; a su vez, dichos sentimientos influyen tanto en el contenido de los pensamientos como en las relaciones que se establecen con dicho objeto. Autores como Castilla del Pino (citado en Asensio, 2002), expresan que la actitud es esencialmente afectiva, emocional y constituye el factor que orienta y gobierna conductas y comportamientos. Con las actitudes se destaca la dimensión afectiva que impregna y orienta la actuación, con lo que se advierte lo que gusta o disgusta al sujeto.

La formación de las actitudes se relaciona estrechamente con la experiencia personal y social de cada individuo, gracias a la interacción que sostiene el sujeto con el entorno (Zaragoza, 2003), la que acontece mediante procesos de imitación o internalización de patrones sociales, prejuicios e influencia cultural (Castro de Bustamante, 2002). De acuerdo con Zabalza (1998), las actitudes se configuran como resultado del proceso de socialización, lo que deviene de la apropiación de patrones cognitivos y conativos del entorno y como producto de la maduración y desarrollo cognitivo del sujeto, en función del conocimiento y experiencia que tenga sobre ellos. Desde esta perspectiva, las actitudes siguen un proceso en paralelo al desarrollo cognitivo.

Katz (1984 citado en Zaragoza, 2003) señala como funciones de las actitudes: la instrumental o utilitaria, mediante la cual una actitud se define en función de sus consecuencias o de la conducta en cuestión, maximizando gratificaciones y minimizando los aspectos desagradables; la expresiva que resalta la capacidad yoica de realización, expresión y autodefinición, en esta se obtienen gratificaciones al expresar actitudes apropiadas al concepto de sí mismo; la egodefensiva que protege a la persona de verdades o situaciones desagradables de sí misma y, por último, la de conocimiento o economía que permite estructurar y organizar el entorno, actuando como esquema o filtro cognitivo.

Existen diferentes modelos explicativos de la actitud; uno de ellos la considera constituida por tres componentes: cognitivo, afectivo y conductual (García et al., 2000; McGuire, 1986). Diversos trabajos (Aguado, Flores \& Alcedo, 2004; Brecker, 
1984) analizan dichos componentes mediante el uso de escalas.

Los trabajos sobre actitudes son amplios y diversos. Se tienen estudios que analizan actitudes hacia las necesidades educativas especiales (Alemany \& Villuendas, 2004; Doménech, Esbrí, González \& Miret, 2004); la discapacidad (Moreno, Rodríguez, Saldaña \& Aguilera, 2006; Shriver, 2003) o programas de cambio de las actitudes (Aguado et al., 2004).

Por su parte, Berry y Dalal (1996 en Parasuram, 1996, p. 234) definen la discapacidad como “(...) tener un impedimento físico o mental (diferencia) que limita una o más actividades de la vida (...) (esto incluye el desarrollo físico, mental y emocional)". Las actitudes hacia la discapacidad se encuentran mediadas tanto por concepciones rígidas y parciales, centradas excesivamente en las restricciones o ausencia de ciertas capacidades, o bien por concepciones más integrales, centradas tanto en el ambiente como en la persona, lo que puede conducir a actitudes más flexibles y de aceptación (García et al., 2000). Doménech et al. (2004) exponen dos enfoques bajo los que se aborda la discapacidad: uno de tipo médico, de influencia estadounidense, que desarrolló el etiquetado y la individualización hacia la discapacidad y el otro, el británico, que se centra más en políticas sociales y educativas.

Diversos estudios constatan el hecho de que las actitudes hacia personas con discapacidad son eminentemente negativas (Avramidis \& Norwich, 2002; Donaldson, 1980; García de la Banda, 1996; Sale \& Carey, 1995; Verdugo \& Arias, 1991), configurando prejuicios y estereotipos que las estigmatizan y las excluyen, lo que tiene un efecto en su calidad de vida (Shriver, 2003).

En el Programa de Acción Mundial para los Impedidos (ONU, 1982), se señala que la percepción de las personas con discapacidad depende de actitudes sociales que condicionan no solamente el trato que las personas no discapacitadas dan a los discapacitados, sino también las propias actitudes de estos (Aguado et al., 2004).

En el estado de Chiapas, se atienden a través de los servicios de educación especial a 7.934 alum- nos (Instituto Nacional de Estadística y Geografía [INEGI], 2007). El objetivo de este estudio es analizar las actitudes que jóvenes y adultos de dicho estado presentan sobre la discapacidad.

\section{Metodología}

El presente trabajo representa el primero de su tipo en el estado de Chiapas. Es exploratorio, no experimental, transeccional y descriptivo. Se aplican dos escalas, mediante las cuales se analizan términos asociados a la discapacidad y actitudes hacia las personas con discapacidad.

\section{Participantes}

La muestra con la que se trabaja la conforman 736 personas, 341 hombres y 395 mujeres. Residen en 3 localidades del estado de Chiapas, una de tipo rural, una ciudad pequeña (menos de 40.000 habitantes) y una urbana (más de 400.000 habitantes).

\section{Instrumentos}

Escala de Valoración de Términos Asociados con la Discapacidad (EVTAD) de Aguado y Alcedo (1999)

Se trata de una escala de tipo Likert de 20 ítems, los cuales son adjetivos comúnmente asociados a discapacidades, que se valoran desde altamente negativos hasta nada negativos. La consistencia interna de la escala, estimada mediante el coeficiente alfa de Cronbach, es de 0.92. Una puntuación más alta para cualquier ítem de la escala refleja una valoración menos negativa hacia las personas con discapacidad.

Escala de Actitudes hacia las Personas con Discapacidad, Forma G (EAPD) de Verdugo, Arias y Jenaro (1994)

Se trata de una escala de evaluación de actitudes ante personas con cualquier tipo de discapacidad, formada por 37 ítems, los cuales integran cinco subescalas: 
1. Valoración de capacidades y limitaciones (VCL) (ítems 1, 2, 4, 7, 8, 16, 21, 29 y 36)

2. Reconocimiento/negación de los derechos (RND) (ítems 6, 9, 12, 13, 14, 15, 22, 23, 27, 35 y 37$)$

3. Implicación personal (IP) (ítems 3, 5, 10, 11, $25,26,31)$

4. Calificación genérica (CG) (ítems 18, 24, 28 y 34)

5. Asunción de roles (AR) (ítems 19, 30 y 33)

La fiabilidad de la escala es de 0.8597. En este trabajo se obtuvo una confiabilidad de 0.886 según el coeficiente alfa de Cronbach. Los ítems que expresan valoración negativa $(1,2,4,5,6,7,8,10$, $15,17,18,23,24,25,26,28,29,31,34,35,37)$ se codificaron de manera inversa en cuanto a los valores que les asigna la escala. Una puntuación más próxima a 1 para cualquier ítem de la escala, refleja una actitud positiva hacia las personas con discapacidad.

Se consideran variables como sexo, edad, estado civil, escolaridad, ocupación y religión de las personas que responden a los instrumentos. Se pregunta también si el encuestado tiene algún tipo de contacto con personas con discapacidad y, en caso afirmativo, la razón de ello (familiar, laboral, asistencial, ocio/amistad u otras razones), su frecuencia (casi permanente, habitual, frecuente o esporádica) y el tipo de discapacidad al que se refiere (física, auditiva, visual, retraso mental o múltiple).

\section{Procedimiento}

La escala fue aplicada en el domicilio de los encuestados. Para ello se dieron las instrucciones tal como se expresan en el instrumento y, posteriormente, se dio inicio al llenado, en el tiempo que cada uno requirió al respecto. Los datos fueron analizados mediante el SPSS, versión 12.0.

\section{Resultados}

Se aplicaron las escalas a un total de 736 sujetos, de los cuales 341 son hombres (46.3\%) y 395 mujeres (53.7 \%). La aplicación se llevó a cabo en tres co- munidades: la ciudad de Tuxtla Gutiérrez con más de 400.00 habitantes $(284 ; 38.6 \%)$; una localidad de menos de 40.000 habitantes, Cintalapa (152; $20.7 \%)$ y una comunidad rural: Pujiltic $(300 ; 40.8$ $\%)$. La edad de los sujetos oscila en un rango de 13 a 83 años; el grupo con mayor número de sujetos es el de 21 a 30 años (268; $36.4 \%)$, seguido del de 31 a $40(164 ; 22.3 \%)$. El estado civil de las personas es en su mayor parte: casado (322; $43.8 \%)$; soltero $(239 ; 32.5 \%)$ y unión libre $(131 ; 17.3 \%)$. El número de sujetos en función del nivel de escolaridad se distribuye proporcionalmente; excepto para aquellos con licenciatura $(230 ; 31.3 \%)$, el resto obtiene porcentajes de entre 13 y $20 \%$ del total. La mayor parte de los sujetos profesa la religión católica (594; 80.7 \%). La ocupación más común es la de ama de casa $(188 ; 25.5 \%)$ seguida de la de estudiante $(174 ; 23.6 \%)$ y la de agricultor (72; $9.8 \%)$.

Del total 312 personas $(42.4 \%)$ refieren haber tenido contacto con personas que presentan una discapacidad, en tanto que 424 (57.6\%) refieren que no. La razón del contacto ha sido, en la mayoría de los casos, de tipo familiar (130; $17.7 \%$ ), la frecuencia del contacto es variable y el tipo de discapacidad más referida es la física $(106 ; 14.4 \%)$ (Tabla1).

En el análisis de los datos de la EAPD, se obtiene lo siguiente. Por subescalas, la de VCL obtiene medias de entre 2.04 a 3.73; la de RND, de 1.82 a 3.93; la de IP de 1.93 a 2.82; la de CG de 3.18 a 3.77 y la de AR, de 2.39 a 2.54 (Tabla 2). Los reactivos que obtienen un puntaje más positivo son:

12. Las personas con discapacidad deberían poder divertirse con las demás personas (RND, Media = 1.82).

14. Las personas con discapacidad deberían poder casarse si lo desean $(\mathrm{RND}$, Media $=1.89)$.

3. Permitiría que su hijo aceptase la invitación a un cumpleaños que le hiciera un niño con discapacidad $($ IP, Media $=193)$.

El tema nodal de estos reactivos hace alusión al derecho a casarse y divertirse de las personas, así como a la convivencia con estos. 
TABLA 1

Datos sociodemográficos

\begin{tabular}{|c|c|c|c|c|}
\hline & \multirow{3}{*}{ Variables } & \multicolumn{2}{|c|}{ Sexo } & \multirow{2}{*}{ Total } \\
\hline & & Hombre & Mujer & \\
\hline & & No. $\%$ & No. $\%$ & No. $\%$ \\
\hline \multirow[t]{3}{*}{ Lugar } & Tuxtla Gutiérrez & $128(17.4)$ & $156(21.2)$ & $284(38.6)$ \\
\hline & Localidades suburbanas & $66(9.0)$ & $86(11.7)$ & $152(20.7)$ \\
\hline & Pujiltic & $147(20.0)$ & $153(20.8)$ & $300(40.8)$ \\
\hline \multirow[t]{6}{*}{ Edad } & 1 (13 a 20 años) & $58(7.9)$ & $70(9.5)$ & $128(17.4)$ \\
\hline & 2 (21 a 30 años) & $124(16.8)$ & $144(19.6)$ & $268(36.4)$ \\
\hline & 3 (31 a 40 años) & $65(8.8)$ & 99 (13.5) & $164(22.3)$ \\
\hline & 4 (41 a 50 años) & $49(6.7)$ & $58(7.9)$ & $107(14.5)$ \\
\hline & 5 (51 a 60 años) & $27(3.7)$ & $20(2.7)$ & $47(6.4)$ \\
\hline & 6 (60 más años) & $18(2.4)$ & $4(0.5)$ & $22(3.0)$ \\
\hline \multirow[t]{5}{*}{ Estado civil } & Soltero & $104(14.1)$ & $135(18.3)$ & $239(32.5)$ \\
\hline & Casado & $154(20.9)$ & $168(22.8)$ & $322(43.8)$ \\
\hline & Divorciado & $9(1.2)$ & $15(2.0)$ & $24(3.3)$ \\
\hline & Viudo & $9(1.2)$ & $11(1.5)$ & $20(2.7)$ \\
\hline & Unión libre & $65(8.8)$ & $66(9.0)$ & $131(17.3)$ \\
\hline \multirow[t]{5}{*}{ Escolaridad } & Ninguno & $71(9.6)$ & $79(10.7)$ & $150(20.4)$ \\
\hline & Primaria & $61(8.3)$ & $83(11.3)$ & $144(19.6)$ \\
\hline & Secundaria & $64(8.7)$ & $52(7.1)$ & $116(15.8)$ \\
\hline & Preparatoria & $41(5.6)$ & $55(7.5)$ & $96(13.0)$ \\
\hline & Licenciatura & $104(14.1)$ & $126(17.1)$ & $230(31.3)$ \\
\hline \multirow[t]{3}{*}{ Religión } & Católica & $281(38.2)$ & $313(42.5)$ & $594(80.7)$ \\
\hline & Protestante & $40(5.4)$ & $59(8.0)$ & $99(13.5)$ \\
\hline & Ninguna & $20(2.7)$ & $23(3.1)$ & $43(5.8)$ \\
\hline \multirow{2}{*}{$\begin{array}{l}\text { Contacto con personas } \\
\text { con discapacidad }\end{array}$} & Sí & $153(20.8)$ & 159 (21.6) & $312(42.4)$ \\
\hline & No & $188(25.5)$ & $236(32.1)$ & $424(57.6)$ \\
\hline \multirow[t]{4}{*}{ Razón del contacto } & Familiar & $52(7.1)$ & $78(10.6)$ & $130(17.7)$ \\
\hline & Laboral & $42(5.7)$ & $18(2.4)$ & $60(8.2)$ \\
\hline & Asistencial & $17(2.3)$ & $15(2.0)$ & $32(4.3)$ \\
\hline & Ocio/amistad & $28(3.8)$ & $36(4.9)$ & $64(8.7)$ \\
\hline
\end{tabular}




\begin{tabular}{llccc} 
Razón del contacto & Otras razones & $14(1.9)$ & $12(1.6)$ & $26(3.5)$ \\
& Sin contacto & $188(25.5)$ & $236(32.1)$ & $424(57.6)$ \\
Frecuencia del contacto & Casi permanente & $39(5.3)$ & $32(4.3)$ & $71(9.6)$ \\
& Habitual & $36(4.9)$ & $47(6.4)$ & $83(11.3)$ \\
& Frecuente & $46(6.3)$ & $50(6.8)$ & $96(13.0)$ \\
& Esporádico & $32(4.3)$ & $30(4.1)$ & $62(8.4)$ \\
Tipo de discapacidad & Sin contacto & $188(25.5)$ & $236(32.1)$ & $425(57.6)$ \\
& Física & $58(7.9)$ & $48(6.5)$ & $106(14.4)$ \\
& Auditiva & $39(5.3)$ & $27(3.7)$ & $66(9.0)$ \\
& Visual & $25(3.4)$ & $30(4.1)$ & $55(7.5)$ \\
& Retraso mental & $20(2.7)$ & $20(2.7)$ & $40(5.4)$ \\
Total & Múltiple & $11(1.5)$ & $34(4.6)$ & $45(6.1)$ \\
& Sin contacto & $188(25.5)$ & $236(32.1)$ & $424(57.6)$ \\
\hline
\end{tabular}

Fuente: elaboración propia.

Por otra parte, los reactivos con mayor puntaje que refieren una actitud más negativa son:

15. Las personas con discapacidad deberían ser confinadas en instituciones especiales (RND, Media $=3.93)$.

34. La mayoría de las personas con discapacidad prefieren trabajar con otras personas que tengan su mismo problema $(\mathrm{CG}$, Media $=3.77)$.

7. Las personas con discapacidad funcionan en muchos aspectos como los niños (VCL, Media = 3.73).

Los temas centrales de estos reactivos se relacionan con la segregación e infantilización de los discapacitados.

De las subescalas, la referida a IP es la que alcanza una media por reactivos más baja (2.42), seguida de la referida a AR (2.48), de la de RND (2.54), en tanto que las más altas son para las de CG (3.40) y la de VCL (3.02) (Tabla 3).

Al comparar las dos muestras en relación con el sexo, se observan diferencias mínimas entre ambos grupos: media de $102.89(\mathrm{DE}=26.64)$ para los hombres, y de $99.29(\mathrm{DE}=26.04)$ para las mujeres.
TABLA 2

Medias por reactivos de EAPD

\begin{tabular}{lcccc}
\hline \multicolumn{1}{c}{ Subescala } & Reactivo & Media & $\begin{array}{c}\text { Media } \\
\text { global }\end{array}$ & $\begin{array}{c}\text { Desviación } \\
\text { estándar }\end{array}$ \\
\hline Valoración de & 1 & 2.64 & 3.02 & 1.76 \\
capacidades y & 2 & 3.43 & & 1.67 \\
limitaciones & 4 & 3.26 & & 1.74 \\
& 7 & 3.73 & 3.02 & 1.66 \\
Valoración de & 8 & 3.15 & & 1.74 \\
capacidades y & 16 & 2.04 & & 1.42 \\
limitaciones & 21 & 2.88 & & 1.41 \\
& 29 & 3.46 & & 1.57 \\
& 36 & 2.62 & & 1.50 \\
Reconoci- & 6 & 2.73 & 2.54 & 1.87 \\
miento/ & 9 & 2.30 & & 1.61 \\
negación de & 12 & 1.82 & & 1.29 \\
los derechos & 13 & 2.26 & & 1.37 \\
& 14 & 1.89 & & 1.27 \\
& 15 & 3.93 & & 1.85
\end{tabular}




\begin{tabular}{|c|c|c|c|c|}
\hline Subescala & Reactivo & Media & $\begin{array}{l}\text { Media } \\
\text { global }\end{array}$ & $\begin{array}{c}\text { Desviación } \\
\text { estándar }\end{array}$ \\
\hline \multirow{11}{*}{$\begin{array}{l}\text { Implicación } \\
\text { personal }\end{array}$} & 22 & 2.23 & \multirow{11}{*}{2.42} & 1.50 \\
\hline & 23 & 2.88 & & 1.70 \\
\hline & 27 & 2.43 & & 1.55 \\
\hline & 35 & 3.16 & & 1.78 \\
\hline & 37 & 2.27 & & 1.68 \\
\hline & 3 & 1.93 & & 1.47 \\
\hline & 5 & 2.82 & & 1.84 \\
\hline & 10 & 2.29 & & 1.72 \\
\hline & 11 & 2.16 & & 1.52 \\
\hline & 25 & 2.44 & & 1.73 \\
\hline & 26 & 2.51 & & 1.70 \\
\hline \multirow{4}{*}{$\begin{array}{l}\text { Calificación } \\
\text { genérica }\end{array}$} & 31 & 2.82 & \multirow{4}{*}{3.40} & 1.93 \\
\hline & 18 & 3.18 & & 1.64 \\
\hline & 24 & 3.26 & & 1.56 \\
\hline & 28 & 3.36 & & 1.62 \\
\hline \multirow{4}{*}{$\begin{array}{l}\text { Asunción de } \\
\text { roles }\end{array}$} & 34 & 3.77 & \multirow{4}{*}{2.48} & 1.63 \\
\hline & 19 & 2.54 & & 1.37 \\
\hline & 30 & 2.39 & & 1.51 \\
\hline & 33 & 2.51 & & 1.49 \\
\hline
\end{tabular}

Fuente: elaboración propia.

TABLA 3

Medias por subescalas de la EAPD

\begin{tabular}{ccccc}
\hline Subescalas & N & Media & $\begin{array}{c}\text { Media por } \\
\text { reactivos }\end{array}$ & $\begin{array}{c}\text { Desviación } \\
\text { estándar }\end{array}$ \\
\hline VCL & 736 & 27.21 & 3.02 & 7.618 \\
RND & 736 & 27.91 & 2.54 & 8.650 \\
IP & 736 & 16.97 & 2.42 & 7.223 \\
CG & 736 & 13.58 & 3.40 & 4.446 \\
AR & 736 & 7.44 & 2.48 & 3.154 \\
\hline
\end{tabular}

Fuente: elaboración propia.

Los resultados en la prueba de Levene muestran que esta no es significativa en el nivel 0.850 , así: $F$ de Levene $=0.036, p>0.05$, por lo que las varianzas no son heterogéneas. Al considerar el estadístico $t=1.850$ se obtuvo un nivel de probabilidad de 0.065 , dado que este no es menor que 0.05, el estadístico $t$ no es significativo, por lo que las medias para hombres y mujeres son iguales.

En la contrastación entre las medias obtenidas en cada una de las variables y el puntaje alcanzado en cada instrumento, se aplicó la prueba post hoc de Tukey para el análisis de contraste, con un nivel de $0.05 \%(p<0.05)$. Respecto al nivel de escolaridad, se obtienen puntajes similares para aquellos que se refieren como sin ningún nivel de escolaridad y primaria. Los grupos sin escolaridad y primaria difieren de aquellos con secundaria, preparatoria y licenciatura. Estos dos últimos también presentan puntajes similares entre sí. Así, aquellos sujetos sin escolaridad y primaria muestran diferencias significativas con aquellos de secundaria, preparatoria y licenciatura, excepto entre estos. Los de secundaria presentan diferencias con todos los demás niveles, en tanto que los de preparatoria con los de sin escolaridad, primaria y secundaria, excepto entre sí. Es relevante considerar que los grupos con más bajo puntaje (actitudes más positivas), se encuentran entre los sujetos de preparatoria y licenciatura, que difieren significativamente de aquellos con menos escolaridad o sin escolaridad.

Los sujetos de los dos primeros grupos de edad (13 a 20 y 21 a 30), no presentan diferencias significativas entre sí, aunque sí con el resto de los grupos de edad, los que a su vez presentan puntajes similares. Destaca que los sujetos más jóvenes presenten actitudes más positivas que el resto de los sujetos, ya que conforme aumenta la edad, la actitud hacia las personas con discapacidad es menos positiva.

El lugar de procedencia de los sujetos presenta un menor puntaje en aquellos de localidades intermedias, muy estrechamente relacionados con los de la ciudad, ambas con diferencias significativas de aquellos de zona rural, que presentan puntajes que señalan actitudes menos positivas.

Los solteros presentan los puntajes más bajos y, conjuntamente a los casados, muestran diferencias significativas de los divorciados, viudos o que viven en unión libre. 
Tanto católicos como protestantes presentan puntajes similares con un intervalo de media menor para los segundos, no obstante, aquellos que no profesan ninguna religión alcanzan los puntajes más bajos, siendo significativa estadísticamente la diferencia entre la media de estos respecto de católicos y protestantes. Así, el no referir una religión, constituye un elemento que deviene en actitudes más positivas hacia personas que presentan una discapacidad.

En los aspectos referidos a la razón y frecuencia del contacto y del tipo de discapacidad de aquellos con quienes han establecido un contacto, se refiere lo siguiente: en cuanto a la razón del contacto, únicamente se aprecian diferencias para aquellos cuya referencia es la familiar de quienes no han tenido contacto con personas con discapacidad, lo que indica actitudes más positivas en quienes tienen un familiar discapacitado.

Aquellos que mantienen un contacto esporádico, son quienes presentan una actitud más positiva hacia las personas con discapacidad. Este grupo presenta diferencias significativas respecto de aquellos que no presentan contacto. Las personas que mantienen contacto con personas con discapacidad múltiple y física, obtienen los puntajes más bajos, cercanos a aquellos con retraso mental, lo que plantea actitudes más positivas en relación con estas discapacidades respecto a otras, como la auditiva y visual.

En el análisis de los datos de la EVTAD, los puntajes en medias de cada uno de los términos referidos en el instrumento, señalan más altos puntajes para los términos de con necesidades educativas especiales, excepcional y enfermo, lo que supone valoración menos negativa, en tanto que los puntajes menores se registran para los términos subnormal, trastornado y retrasado (Tabla 4).

Al comparar las dos muestras con relación al sexo, se observan diferencias mínimas entre ambos grupos: media de $50.58(D E=16.39)$ para los hombres y de 50.21 ( $D E=16.78)$ para las mujeres.

En la prueba de Levene los resultados muestran que esta no es significativa en el nivel 0.787 , así: $F$ de Levene $=0.073, p>0.05$, por lo que las varianzas no son heterogéneas. Al considerar el
TABLA 4

Medias y desviación estándar de los términos de la EVTAD

\begin{tabular}{lccc}
\hline & Media & Desviación estándar & $N$ \\
Marginado & 2.18 & 1.255 & 736 \\
Retrasado & 2.13 & 1.179 & 736 \\
Transtornado & 2.10 & 1.199 & 736 \\
Excepcional & 3.38 & 1.411 & 736 \\
Lisiado & 2.50 & 1.310 & 736 \\
Tullido & 2.42 & 1.341 & 736 \\
Minusválido & 3.02 & 1.237 & 736 \\
Con ne. & 3.51 & 1.367 & 736 \\
Enfermo & 3.06 & 1.280 & 736 \\
Anormal & 2.28 & 1.290 & 736 \\
Deficiente & 2.25 & 1.231 & 736 \\
Inadaptado & 2.24 & 1.303 & 736 \\
Inválido & 2.47 & 1.310 & 736 \\
Discapacitado & 2.93 & 1.502 & 736 \\
Incapacitado & 2.80 & 1.378 & 736 \\
Desvalido & 2.39 & 1.317 & 736 \\
Disminuido & 2.35 & 1.318 & 736 \\
Mutilado & 2.15 & 1.360 & 736 \\
Subnormal & 2.07 & 1.285 & 736 \\
Impedido & 2.15 & 1.401 & 736 \\
\hline Fuer & &
\end{tabular}

Fuente: elaboración propia.

estadístico $t=0.306$, se obtuvo un nivel de probabilidad de 0.760 , dado que este no es menor que 0.05 , el estadístico $t$ no es significativo, por lo que las medias para hombres y mujeres son iguales.

En cuanto a la contrastación entre las medias de los sujetos según el nivel de escolaridad, se obtienen puntajes similares para aquellos que se refieren sin ningún nivel de escolaridad y primaria y más alto para aquellos con estudios de preparatoria y licenciatura, lo que señala una valoración menos negativa de los términos referidos para estos últimos. Los grupos de sin escolaridad y primaria $(p<$ 
0.05), difieren de aquellos con secundaria, preparatoria y licenciatura. Estos dos últimos también presentan puntajes similares entre sí. Así, aquellos sujetos sin escolaridad y primaria muestran diferencias significativas con aquellos de secundaria, preparatoria y licenciatura, excepto entre estos. Los de secundaria presentan diferencias con todos los demás niveles, menos con los de preparatoria.

Los sujetos de los dos primeros grupos de edad (13 a 20 y 21 a 30), presentan diferencias significativas con los sujetos del grupo 4 (41 a 50 años), quienes obtienen la media más baja. No se aprecian diferencias significativas entre el resto de los grupos de edad. Destaca que los sujetos más jóvenes presenten una valoración menos negativa de los términos referidos en la escala.

El lugar de procedencia de los sujetos presenta un menor puntaje en aquellos de localidad rural respecto de los de localidades intermedias y los de la ciudad, quienes presentan puntajes muy similares entre sí, ambas con diferencias significativas de aquellos de zona rural, que muestran puntajes que señalan una valoración menos negativa de los términos asociados a la discapacidad.

Los solteros presentan los puntajes más bajos, con diferencias en relación con los casados, divorciados o que viven en unión libre. Los casados, a su vez, muestran diferencias con los divorciados o que viven en unión libre. El rango de valores mínimo y máximo de los viudos es tan amplio que no conlleva a diferencias significativas con ninguno de los grupos.

La variable religión no presenta diferencias significativas entre católicos, protestantes y sin ninguna religión, aunque la media es mayor para estos últimos.

En los aspectos referidos a la razón y frecuencia del contacto y del tipo de discapacidad de aquellos con quienes han establecido un contacto, se refiere lo siguiente: en cuanto a la razón del contacto, no se aprecian diferencias estadísticamente significativas entre las distintas variables, aunque la media es ligeramente mayor para aquellos cuya referencia es la familiar y otras razones.

En cuanto a la frecuencia del contacto, tampoco se evidencian diferencias entre las distintas variables de estudio, aunque la media es ligeramente más alta para la opción de contacto esporádico. Las personas que mantienen contacto con personas con discapacidad física obtienen los puntajes más altos, cercanos a aquellos con retraso mental o múltiple, aunque la diferencia solo es estadísticamente significativa entre la variable física y la visual.

\section{Conclusiones}

En la aplicación de la EAPD la subescala IP alcanza puntajes más cercanos a 1 , lo que sugiere actitudes más positivas. En esta se incluyen situaciones relacionadas con la participación con personas con discapacidad, así, parece existir mayor disposición a un acercamiento social. Del mismo modo, en la subescala $\mathrm{AR}$, en que se incluyen reactivos relacionados con la valoración que los sujetos con discapacidad tienen de sí mismos, también resulta favorable, al igual que en la subescala RND. Estos resultados son claves para la inserción social de las mismas. No obstante, lo obtenido en la subescala VCL, relacionada con la competencia cognitiva, laboral y social, resulta ser poco alentador. Sobre este tema, el trabajo de Ammerman (1997) señala cómo los prejuicios hacia estas personas, llevan a verlas como inferiores, incompetentes e incluso infantiles. En la subescala CG, que incluye la presencia de malhumor, desconfianza, resentimiento o timidez, el puntaje alcanzado refleja actitudes menos positivas, así, se considera como uno de los aspectos que se considera menos desarrollado en personas con discapacidad.

No se advierten diferencias entre las calificaciones de hombres y mujeres, no obstante, variables como una mayor escolaridad, el ser más joven (de 13 a 30 años), el ser soltero o casado, vivir en zona urbana y no profesar ninguna religión, constituyen aspectos que señalan actitudes más positivas hacia las personas con discapacidad. Estos indicadores son similares en los resultados obtenidos tanto en la EAPD y la EVTAD, aunque en esta última, en la variable edad, las diferencias son significativas solo entre los sujetos de entre 13 a 30 años, respecto de aquellos del rango 41 a 50, no así con el resto. La variable religión no resulta significativa entre los 
distintos grupos de análisis. Resultados similares son obtenidos en otros estudios como el de Parasuram y Shreyas (2006) en la India y Moreno et al. (2006) en España.

Por otra parte, tener un familiar que presente alguna discapacidad, constituye un elemento que conlleva a una mayor sensibilidad y actitud más positiva hacia estos, aspecto igualmente enfatizado en el estudio de Moreno et al. (2006), aunque opuesto a lo encontrado en el trabajo de Parasuram y Shreyas (2006) quienes niegan esta relación; asimismo, el contacto esporádico, con estas personas hace que esta actitud se refuerce. Desafortunadamente, al parecer, aquellos que mantienen un contacto más permanente, presentan actitudes menos positivas hacia estos, lo que también puede ser indicativo del cansancio o malestar que deviene de una atención más estrecha. Las personas con discapacidades hacia quienes se presenta una actitud más positiva, son aquellas con una discapacidad múltiple, física o de retraso mental, y menor en aquellos con discapacidad visual o auditiva. Ello puede indicar una situación de mayor consideración en cuanto más evidente y limitante sea la discapacidad. En la EVTAD no existen diferencias significativas entre los sujetos que señalen una u otra opción de razón del contacto hacia personas con discapacidad; se coincide, además, en que en aquellos que mantienen una frecuencia esporádica de relación, refieran una valoración menos negativa de los términos, en tanto que respecto al tipo de discapacidad, solo se aprecian diferencias significativas en la valoración de términos a personas con discapacidades física y visual.

Con base en lo anteriormente expuesto, se puede señalar que en las nuevas generaciones su menor edad, su mayor escolarización y su condición de soltería o casados, son variables importantes para el desarrollo de actitudes positivas hacia las personas que presentan una discapacidad. Zabalza (1998) argumenta en torno a la relación con el desarrollo cognitivo, como mucho más acode con la escolarización y las actitudes de las personas. Estas variables adquieren mayor importancia, si se consideran tales condiciones en zonas urbanas respecto de las rurales. Chiapas es un estado con más de 70 localidades con población similar a aquella en la que se realiza el estudio y con más de 19.000 con menos de 500 habitantes (INEGI, 2007). El reto es mayor si se pretende una mayor atención a personas que presenten una discapacidad en estas localidades, ya que es de esperarse actitudes menos favorables, más parciales y rígidas para estos. La experiencia social -los aprendizajes que la cultura impone- constituye un aliciente o un abismo con el cual se tiene que trabajar para un desarrollo de actitudes (Zaragoza, 2003), situación inicial que se orienta posteriormente a una mayor integración e inclusión de los sujetos que presentan una discapacidad (Alemany \& Villuendas, 2004; Shriver, 2003).

Las actitudes de los encuestados que conocen personas con discapacidades son más positivas que las de los que no tenían conocimiento de ellas. La condición de empatía y acercamiento afectivo es, sin duda, un aspecto que deberá observarse en programas de intervención, los resultados en este estudio son coincidentes con diversos trabajos sobre el tema (Aguado et al., 2004; Asensio, 2002; Avramidis \& Norwich, 2002). Así, el contacto con las personas con discapacidad aumenta las actitudes positivas y se constituye en una variable clave en la configuración de las actitudes para con ellos (Strohmer, Grand \& Purcell, 1984).

\section{Referencias}

Aguado, A. L. \& Alcedo, M. A. (1999). La Escala de Valoración de Términos Asociados a la Discapacidad en una muestra de EGB. Análisis y Modificación de Conducta, 25 (103), 783-806.

Aguado, A. L., Flores, M. A. \& Alcedo, M. A. (2004). Programas de cambios de actitudes ante la discapacidad. Psicothema, 16 (4), 663-673.

Alemany, I. \& Villuendas, M. D. (2004). Las actitudes del profesorado hacia el alumnado con necesidades educativas especiales. Convergencia, 11 (34), 183-215.

Ammerman, R. T. (1997, marzo). Nuevas tendencias en investigación sobre discapacidad. Conferencia en las II Jornadas Científicas de Investigación sobre Personas con Discapacidad, Salamanca, España. 
Asensio, J. M. (2002). Las actitudes en la reforma: un aspecto de la educación emocional. Revista Española de Pedagogía, 221, enero-abril, 51-64.

Avramidis, E. \& Norwich, B. (2002). Teachers' attitudes toward integration/inclusion: A review of the literature. European Journal of Special Needs Education, 17 (2), 129-147.

Brecker, J. (1984). Empirical validation of affect, behavior and cognition as distinct components of attitude. Joumal of Personality and Social Psychology, 47, 1191-1205.

Castro de Bustamante, J. (2002). Análisis de los componentes actitudinales de los docentes hacia la enseñanza de la matemática: caso: $1^{\circ}$ y $2^{\circ}$ Etapas de Educación Básica. Municipio San Cristóbal-Estado Táchira. Tesis doctoral en Pedagogía, Departament de Pedagogia, Universitat Rovira i Virgili, Tarragona, España.

Doménech, V., Esbrí, J. V., González, H. A. \& Miret, L. (2004). Actitudes del profesorado hacia el alumnado con Necesidades Educativas Especiales Derivadas de Discapacidad. Novenes Jornades de Foment de la Investigació, Universitat Jaume I, Castelló, España.

Donaldson, J. (1980). Changing attitudes toward handicapped persons: A review and analysis of research. Exceptional Children, 46 (7), 504-514.

Dorsch, F. (2005). Diccionario de Psicología. Barcelona: Herder.

García, I., Escalante, I., Escandón, M. C., Fernández, L. G., Mustri Dabbah, A. \& Puga, I. (2000). La integración educativa en el aula regular. Principios finalidades y estrategias. México: SEP/Fondo Mixto México/España.

García de la Banda, G. \& Pelechano, V. (1996). Variables de personalidad como determinantes de las actitudes de aceptación y rechazo de invidentes en padres y profesores. Análisis y Modificación de Conducta, 22 (81), 5-36.

Instituto Nacional de Estadística y Geografía. (2007). Anuario Estadístico del Estado de Chiapas (Vol. 1). Tuxtla Gutiérrez, Chiapas: Autor.

McGuire, W. J. (1986). Vicissitudes of attitudes and similar representational constructs in twentieth century Psychology. Eur. J. Soc. Psicol., 16, 89 -130 .
Moreno, F. J., Rodríguez, I., Saldaña, D. \& Aguilera, A. (2006). Actitudes ante la discapacidad en el alumnado universitario matriculado en materias afines. Revista Iberoamericana de Educación, 40, 1-12.

Organización de Naciones Unidas. (1982). Programa de Acción Mundial para los Impedidos. A.G. Res. 37/52. 37a sesión. Supp. No. 51. Doc. A/37/51. (diciembre 3, 1982).

Parasuram, K. \& Shreyas, T. (2006). Variables that affect teachers' attitudes towards disability and inclusive education in Mumbai, India. Disability \& Society, 21 (3), 231-242.

Real Academia Española. (2001). Diccionario de la Lengua Española (22⿳亠丷. ed.). Madrid: Espasa-Calpe.

Sale, P. \& Carey, D. M. (1995). The sociometric status of students with disabilities in a full-inclusion school. Exceptional Children, 62, 6-19.

Shriver, T. (2003, junio). Estudio Multinacional de Actitudes hacia las personas con discapacidad intelectual. Resultados Generales y Llamada a la Acción (Informe del Comité de Olimpiadas Especiales). Washington/Boston: Special Olympics/Center for Social Development and Education.

Strohmer, D. C., Grand, S. A. \& Purcell, M. J. (1984). Attitudes toward persons with disability: An examination of demographic factors, social context and specific disability. Rehabilitation Psychology, 29, 131-145.

Verdugo, M. A. \& Arias, B. (1991). Evaluación y modificación de las actitudes hacia los minusválidos. Revista de Psicología General y Aplicada, 44 (1), 95-102.

Verdugo, M. A., Arias, B. \& Jenaro, C. (1994). Actitudes hacia las personas con minusvalía. Madrid: Instituto Nacional de Servicios Sociales.

Zabalza, M. A. (1998). Evaluación de actitudes y valores. En A. Medina, J. Cardona, S. Castillo \& M. C. Domínguez (Eds.), Evaluación de los procesos y resultados de aprendizaje de los estudiantes. Madrid: Editorial UNED.

Zaragoza, J. M. (2003). Actitudes del profesorado de secundaria obligatoria hacia la evaluación de los aprendizajes de los alumnos. Tesis Doctoral, Departamento de Pedagogía Aplicada, Facultad de Ciencias de la Educación, Universidad Autónoma de Barcelona, España. 
